\title{
Congenital malformations in Ecuadorian children: urgent need to create a National Registry of Birth Defects
}

This article was published in the following Dove Press journal:

The Application of Clinical Genetics

13 April 2010

Number of times this article has been viewed

\author{
Fabricio González-Andrade' \\ Ramiro López-Pulles ${ }^{2}$ \\ 'Department of Medicine, \\ Metropolitan Hospital, Quito, \\ Ecuador; ${ }^{2}$ Science and Technology \\ Process of the Ministry of Public \\ Health, Biomedical Center of the \\ Central University and Regional \\ Autonomic University of the Audes, \\ Quito, Ecuador Universidad Central \\ del Ecuador, Quito, Ecuador
}

Aim: This study sets out (a) to estimate the prevalence of admissions by birth defects, using the official database of hospitals of Ecuador; and (b) to set the basis for a new National Register of Birth Defects in Ecuador that works as a program for the clinical and epidemiological investigation of risk factors in the etiology of congenital anomalies in Ecuadorian hospitals, using a case-control methodological approach. This is the first report in their class.

Methods: The data used in this study are derived from the National Register of Hospital Admission/Discharges of the Instituto Nacional de Estadísticas y Censos; data of the Ministry of Public Health were also used. Ecuador does not have an official Medical Birth Registry or a Congenital Malformations Registry.

Results: A total of 51,375 discharges by congenital malformations were registered in a 7-year period. Of these, 16,679 admissions were of children aged less than 1 year of age, with a birth prevalence rate (BPR) of 72.33/10,000 births. $77 \%$ of the congenital defects registered comprise the 50 most common birth defects observed in this age group. Cleft lip was the most prevalent birth defect in children less than 1 year of age and the second most common defect in children 1 to 5 years of age. Unilateral cleft lip shows a BPR of 4.57/10,000 births; cardiac birth defects as a group have a BPR of 4.2; hydrocephalus a BPR of 3.77; and Down's syndrome a BPR of 3.70. Undescended testicle was the most prevalent birth defect in children between 1 to 5 years. 9384 children under 1 year of age were male $(55.9 \%)$ and 7053 were female $(42.1 \%)$. BPR in males was 40.45 and in females 30.40 .

Conclusion: This report documents the prevalence estimates for birth defects reported in the hospital discharge data. These estimates are important to 1) plan for health-care and education needs of the Ecuadorian population, 2) identify increased occurrences of birth defects in specific geographic regions, 3) serve as a reference point for assessment of provincial surveillance systems, 4) evaluate national public health interventions, 5) compare Ecuador prevalence estimates with those of other countries, and 6) help determine the appropriate allocation of resources for basic and public health research. There is an urgent need to establish a National Registry of Birth Defects involving different sources of information such as prenatal medical records, birth records and medical records during the first year of life at an early stage, and surveys on cytogenetic prenatal diagnostic surveys and cytogenetics of therapeutic abortions.

Keywords: Ecuador, genetics, birth defects surveillance, database, prevalence, epidemiology, congenital malformations

\section{Introduction}

This study sets out (a) to estimate the prevalence of admissions by birth defects, using the database of hospitals of Ecuador; and (b) to set the basis for a new National Register of Birth Defects in Ecuador that works as a program for the clinical and 
epidemiological investigation of risk factors in the etiology of congenital anomalies in Ecuadorian hospitals, using a case-control methodological approach. This is the first report in their class.

There is a lack of information on population-based studies of malformations in Ecuador; ${ }^{1}$ the surveillance of the effects of prenatal screening and diagnosis on the birth prevalence of malformations is limited by gaps and wide variations in surveillance systems. ${ }^{2}$ This justifies the use of official databases in the production of the information only as an initial step in the development of an organized and generalized system that gathers in an organized way birth defects data, as proposed in this paper.

The only official existing data are limited to a few hospitals, such as those that are part of the ECLAMC network (Latin American Collaborative Study on Congenital Malformations). ${ }^{3}$ This network was established in 1973 , originally with 12 maternity hospitals in 6 provinces around the country, covering approximately $4.16 \%$ of the total number of live births in Ecuador. This network reported $1.7 \%$ of newborns $(1,114 / 66,843)$ and $1.3 \%$ of stillbirths $(891 / 66,843)$ with some type of congenital malformation, in a study made between June 2001 and June 2005. ${ }^{4}$ The hospitals associated with this network are mostly secondary care hospitals located in the cities of Quito, Manta, Chone, Bahía de Caráquez, Portoviejo, Cañar, Azogues, Ibarra, Loja and Machala. ${ }^{5}$ Despite the importance of this network, this study did not cover the whole country because participation is still voluntary, which limits coverage to less of $5 \%$ of all live births in Ecuador. However, morbidity studies of children indicated that genetic diseases and congenital malformations represent $10 \%$ to $25 \%$ of hospitalizations in tertiary care facilities, especially in some Latin America cities. ${ }^{6,7}$

Ecuador has a relatively small but genetically highly diverse population across the whole country. There is a need for a central coordination of information on availability of prenatal diagnosis, newborn screening, and genetic testing with modern genetic technologies. It should be stressed that demographic factors also have a great influence on the prevalence of some congenital malformations. ${ }^{8}$ In this regard, it should be noted that Ecuador is located in western South America, ${ }^{9}$ where half the population lives in cities with altitudes of $2000 \mathrm{~m}$ asl. ${ }^{10}$ The population is 14 million, approximately half (54\%) of whom live in urban zones, especially in the four largest cities of Quito, Guayaquil, Cuenca and Santo Domingo. ${ }^{11}$ Ecuador is a multi-ethnic country with a strong Native Amerindian culture ${ }^{12}$ that coexists with Mestizo and Afroamerican ethnic groups.
Childbearing-age women in Ecuador are potentially exposed to high teratogenic risks such as infectious agents, environmental chemical compounds, radiation, drugs, and maternal metabolic diseases. ${ }^{13}$ These risk factors interact with the low levels of school education and low socioeconomic status of the population, and scarce resources in the public health care system for the prevention and treatment of congenital anomalies. ${ }^{14}$ The birth prevalence of congenital anomalies in developing countries is underestimated, mainly due to deficiencies in diagnostic capabilities and lack of reliability of medical records and health statistics. ${ }^{8}$ The recorded rates in developing countries must be considered minimum estimates because of low diagnostic capacities and underreporting. ${ }^{8}$

According to the health authorities of the United States, ${ }^{15}$ birth defects are a significant public health concern, affecting $\sim 3 \%$ of all births, and resulting in many elective pregnancy terminations or spontaneous abortions, a situation similar to that seen in Europe, where birth defects affect $\sim 2 \%$ of all births. ${ }^{16}$ Every year, $\sim 150,000$ babies are born with birth defects in developed countries. ${ }^{17}$ Birth defects and genetic diseases are the leading causes of infant deaths, resulting in substantial mortality and morbidity throughout childhood. ${ }^{18}$ Although birth defects account for only $15 \%$ to $30 \%$ of all pediatric hospitalizations in developed countries, they have a proportionally higher cost in health care programs than other hospitalizations. ${ }^{19}$ In the US, US $\$ 8$ billion are spent each year to provide medical and rehabilitative care for affected children. Thus, birth defects impart a significant burden to families and society. ${ }^{20}$

The causes of around $40 \%$ to $50 \%$ of birth defects are unknown, ${ }^{21} 20 \%$ are attributed to a combination of hereditary and environmental factors, $8 \%$ to $10 \%$ to single-gene mutations, $6 \%$ to chromosome abnormalities, and 5\% to maternal illnesses. ${ }^{21}$ There is also an important increase in birth defects and developmental disabilities due to maternal conditions. $^{22}$ These maternal conditions comprise pregestational diabetes and phenylketonuria, and other related pathologies. Maternal diabetes mellitus is associated with increased teratogenesis, which can occur in pre-gestational diabetes type 1 and type 2 . Cardiac defects and neural tube defects are the most common malformations observed in fetuses of pre-gestational mothers with diabetes. ${ }^{23}$

Certain infections, such as rubella virus (which causes German measles and congenital rubella syndrome) and cytomegalovirus (which causes a congenital infection that also produces birth defects and developmental disabilities), have shown to be highly teratogenic. ${ }^{24}$ Equally, some medications as valproic acid and isotretionin have shown 
teratogenic effects. The most teratogenic anti-epileptic drug seems to be valproic acid which causes about $2 \%$ of neural tube diseases and an additional increase of $4 \%$ to $8 \%$ in major congenital anomalies. ${ }^{25}$ In this description, lifestyle factors such as alcohol consumption and smoking are also considered as teratogenic. Alcohol causes fetal alcohol syndrome and other spectrum disorders, for which strong prevention strategies have been developed. ${ }^{26}$ All of the above-mentioned causes are preventable risk factors. ${ }^{27}$

\section{Material and methods Design}

This is an observational descriptive study that evaluates the epidemiological issues of birth defects reported in Ecuador between the years of 2001 and 2007.

\section{Source of information}

The data used in this study were taken from the National Register of Hospital Admission/Discharges of the Instituto Nacional de Estadísticas y Censos (INEC), ${ }^{17}$ and the Ministry of Public Health (MSP). ${ }^{28}$ The Register of Admissions/Discharges is national and funded by the government. The information from the register is provided primarily by the centers and hospitals belonging to the MSP which has a national coverage and includes public maternity clinics, children's hospitals, and general adult hospitals. The information in this register was gathered from the individual clinic history, by a clerk who codes the diagnosis. This register has been, formerly and extensively, used in epidemiological studies. ${ }^{8}$ Ecuador does not have an official Medical Birth Registry or a Congenital Malformations Registry.

\section{Study variables}

Major congenital malformation was defined as a defect detected at birth, delivery or during the first year of life, which results in death (including still birth); it requires major surgery, or has a major effect on the quality of life for the child. In this study, we used the International Classification of Diseases, 10th Edition of 1997 (ICD-10) to classify the Congenital Malformations, Deformations, and Chromosomal Abnormalities, all included in the Q00 to Q99 codes. ${ }^{29}$ The data of two main groups of children, under the age of 1 year, and 1 to 5 years of age, were analyzed. All remaining age groups were excluded because it could not be identified if the patient was to be admitted by walk-ins or subsequent appointment. As an additional measure, duplicate records were excluded from the database and whether each patient was registered only once was reviewed, for the readmissions registry.

\section{Statistical analysis}

We analyzed birth prevalence rate (BPR) by 10,000 births. We also used data on admissions/discharges of children under 1 year of age who were admitted by birth defects or other previously acquired related complications.

\section{Results}

A total of 51,375 discharges by congenital malformations were registered in Ecuador, during the 7-year period analyzed, for all age groups. Out of the total, 16,679 admissions were of children less than 1 year of age, and with a BPR of $72.33 / 10,000$ births. Seventy-seven percent of these comprised the 50 most common birth defects observed in this age group (see Table 1). The most common birth defect found is the unilateral cleft lip with a BPR of 4.57/10,000 births, followed by congenital heart malformations with a BPR 4.2; hydrocephalus with a BPR of 3.77 and Down's syndrome with a BPR of 3.70. 9384 children under 1 year of age were male $(55.9 \%)$ and 7053 were female $(42.1 \%)$. The BPR of males was 40.45 and of females 30.40 .

Table I The most common birth defects in children under I year of age admitted to Ecuadorian hospitals

\begin{tabular}{|c|c|c|c|c|c|c|c|c|c|c|c|c|}
\hline Rank & Code & Description & 2001 & 2002 & 2003 & 2004 & 2005 & 2006 & 2007 & Total & $\%$ & BPR \\
\hline I & Q369 & Cleft lip, unilateral & 208 & 195 & 137 & 112 & 143 & 138 & 126 & 1059 & 6.35 & 4.57 \\
\hline 2 & Q249 & Congenital malformation of heart, unspecified & $|3|$ & 119 & 143 & 177 & 139 & 132 & 135 & 976 & 5.85 & 4.21 \\
\hline 3 & Q039 & Hydrocephalus & 113 & 0 & 163 & 130 & 173 & 135 & 161 & 875 & 5.25 & 3.77 \\
\hline 4 & Q909 & Down's syndrome & 109 & 137 & 127 & 127 & 122 & 134 & 102 & 858 & 5.14 & 3.70 \\
\hline 5 & Q250 & Patent ductus arteriosus & 68 & 95 & 55 & 69 & 68 & 95 & 57 & 507 & 3.04 & 2.19 \\
\hline 6 & Q059 & Spina bifida, unspecified & 88 & 117 & 64 & 53 & 66 & 55 & 54 & 497 & 2.98 & 2.14 \\
\hline 7 & Q359 & Cleft palate, unilateral & 71 & 64 & 71 & 54 & 45 & 67 & 70 & 442 & 2.65 & 1.91 \\
\hline 8 & Q400 & Congenital hypertrophic pyloric stenosis & 62 & 66 & 36 & 53 & 66 & 61 & 64 & 408 & 2.45 & 1.76 \\
\hline 9 & Q750 & Craniosynostosis & 38 & 34 & 40 & 78 & 62 & 61 & 90 & 403 & 2.42 & 1.74 \\
\hline
\end{tabular}


Table I (Continued)

\begin{tabular}{|c|c|c|c|c|c|c|c|c|c|c|c|c|}
\hline Rank & Code & Description & 2001 & 2002 & 2003 & 2004 & 2005 & 2006 & 2007 & Total & $\%$ & BPR \\
\hline 10 & Q899 & Congenital malformation, unspecified & 39 & 24 & 59 & 113 & 68 & 70 & 24 & 397 & 2.38 & I.7I \\
\hline 11 & Q205 & Discordant atrioventricular connection & 95 & 108 & 37 & 38 & 33 & 30 & 49 & 390 & 2.34 & 1.68 \\
\hline 12 & Q379 & Unspecified cleft palate with unilateral cleft lip & 39 & 56 & 51 & 57 & 71 & 61 & 31 & 366 & 2.19 & 1.58 \\
\hline 13 & Q423 & Imperforate anus & 47 & 58 & 38 & 37 & 24 & $4 \mathrm{I}$ & 40 & 285 & 1.71 & 1.23 \\
\hline 14 & Q539 & Undescended testicle, unspecified & 33 & 26 & 37 & 29 & 57 & 59 & 39 & 280 & 1.68 & 1.21 \\
\hline 15 & Q 170 & Microtia & 6 & 26 & 32 & 51 & 40 & 50 & 71 & 276 & 1.65 & 1.19 \\
\hline 16 & Q439 & Congenital malformation of intestine, unspecified & 32 & 39 & 38 & 38 & 38 & 46 & 42 & 273 & 1.64 & 1.18 \\
\hline 17 & Q663 & Other congenital varus deformities of feet & 60 & 32 & 30 & 27 & 31 & 41 & 51 & 272 & 1.63 & 1.17 \\
\hline 18 & Q699 & Polydactyly, unspecified & 39 & 39 & 27 & 31 & 40 & 46 & 36 & 258 & 1.55 & I.II \\
\hline 19 & Q652 & Congenital luxation of the hip & 21 & 42 & 37 & 36 & 22 & 29 & 37 & 224 & 1.34 & 0.97 \\
\hline 20 & Q390 & Atresia of esophagus without fistula & 26 & 43 & 25 & 25 & 36 & 33 & 27 & 215 & 1.29 & 0.93 \\
\hline 21 & Q212 & Atrioventricular septal defect & 10 & 15 & 18 & 20 & 27 & 82 & 33 & 205 & 1.23 & 0.88 \\
\hline 22 & Q999 & Chromosomal abnormality, unspecified & 11 & 16 & 9 & 33 & 30 & 103 & 3 & 205 & 1.23 & 0.88 \\
\hline 23 & Q043 & Other reduction deformities of brain & 8 & 38 & 35 & 32 & 37 & 22 & 25 & 197 & 1.18 & 0.85 \\
\hline 24 & Q38I & Ankyloglossia & 20 & 15 & 21 & 25 & 19 & 34 & 53 & 187 & 1.12 & 0.81 \\
\hline 25 & Q793 & Gastroschisis & 9 & 14 & 32 & 29 & 29 & 43 & 26 & 182 & 1.09 & 0.78 \\
\hline 26 & Q248 & Other specified congenital malformations of heart & 15 & 33 & 24 & 13 & 11 & 65 & 9 & 170 & 1.02 & 0.73 \\
\hline 27 & Q668 & Clubfoot NOS (not otherwise specified) & 24 & 19 & 21 & 26 & 32 & 20 & 21 & 163 & 0.98 & 0.70 \\
\hline 28 & Q213 & Tetralogy of Fallot & 17 & 32 & 16 & 9 & 29 & 25 & 21 & 149 & 0.89 & 0.64 \\
\hline 29 & Q658 & Other congenital deformities of hip & 17 & 22 & 15 & 31 & 12 & 32 & 16 & 145 & 0.87 & 0.63 \\
\hline 30 & Q419 & $\begin{array}{l}\text { Congenital absence, atresia and stenosis } \\
\text { of small intestine, part unspecified }\end{array}$ & 13 & 21 & 29 & 17 & 25 & 27 & 12 & 144 & 0.86 & 0.62 \\
\hline 31 & Q21I & Atrial septal defect & 0 & 12 & 10 & 23 & 24 & 34 & 35 & 138 & 0.83 & 0.59 \\
\hline 32 & Q02 & Microcephaly & 6 & 14 & 22 & 28 & 16 & 16 & 36 & 138 & 0.83 & 0.59 \\
\hline 33 & Q660 & Talipes equinovarus & 10 & 25 & 19 & 11 & 18 & 9 & 36 & 128 & 0.77 & 0.55 \\
\hline 34 & Q019 & Encephalocele & 7 & 15 & 14 & 14 & 14 & 29 & 18 & 111 & 0.67 & 0.48 \\
\hline 35 & Q790 & Congenital diaphragmatic hernia & 15 & 10 & 16 & 13 & 20 & 13 & 22 & 109 & 0.65 & 0.47 \\
\hline 36 & Q410 & $\begin{array}{l}\text { Congenital absence, atresia and stenosis } \\
\text { of duodenum }\end{array}$ & 13 & 11 & 12 & 9 & 15 & 17 & 27 & 104 & 0.62 & 0.45 \\
\hline 37 & Q203 & Transposition of great vessels & 18 & 17 & 12 & 12 & 10 & 19 & 11 & 99 & 0.59 & 0.43 \\
\hline 38 & $\mathrm{Q} 212$ & Atrioventricular septal defect & 0 & 23 & 10 & 16 & 14 & 17 & 16 & 96 & 0.58 & 0.41 \\
\hline 39 & Q897 & $\begin{array}{l}\text { Multiple congenital malformations, } \\
\text { not elsewhere classified }\end{array}$ & 11 & 12 & 15 & 18 & 9 & 20 & 9 & 94 & 0.56 & 0.41 \\
\hline 40 & Q03। & Atresia of foramina of Magendie and Luschka & 33 & 21 & 12 & 15 & 0 & 7 & 0 & 88 & 0.53 & 0.38 \\
\hline 41 & Q792 & Exomphalos & 9 & 12 & 15 & 11 & 13 & 17 & 11 & 88 & 0.53 & 0.38 \\
\hline 42 & Q228 & $\begin{array}{l}\text { Other congenital malformations } \\
\text { of tricuspid valve }\end{array}$ & 10 & 13 & 25 & 10 & 13 & 11 & 5 & 87 & 0.52 & 0.38 \\
\hline 43 & Q43I & Hirschsprung's disease & 17 & 9 & 15 & 7 & 10 & 11 & 16 & 85 & 0.51 & 0.37 \\
\hline 44 & Q620 & Congenital hydronephrosis & 7 & 15 & & 17 & 14 & 21 & 9 & 83 & 0.50 & 0.36 \\
\hline 45 & Q054 & Unspecified spina bifida with hydrocephalus & 15 & 14 & 13 & 8 & 13 & 3 & 15 & 81 & 0.49 & 0.35 \\
\hline 46 & Q000 & Anencephaly & 10 & 11 & 12 & 14 & 17 & 8 & 8 & 80 & 0.48 & 0.34 \\
\hline 47 & Q378 & Unspecified cleft palate with bilateral cleft lip & 7 & 8 & 19 & 13 & 7 & 11 & 7 & 72 & 0.43 & 0.31 \\
\hline 48 & Q224 & Congenital tricuspid stenosis & 16 & 11 & 10 & 8 & 13 & 7 & 6 & 71 & 0.43 & 0.31 \\
\hline 49 & Q223 & $\begin{array}{l}\text { Other congenital malformations } \\
\text { of pulmonary valve }\end{array}$ & 2 & 0 & 0 & 0 & 19 & 44 & 2 & 67 & 0.40 & 0.29 \\
\hline \multirow[t]{4}{*}{50} & Q255 & Atresia of pulmonary artery & 12 & 13 & 11 & 8 & 6 & 8 & 7 & 65 & 0.39 & 0.28 \\
\hline & & Subtotal & 1687 & 1811 & 1729 & 1825 & 1860 & 2159 & $|82|$ & 12892 & 77 & 55.58 \\
\hline & & Remain birth defects & 460 & 702 & 511 & 442 & 624 & 605 & 543 & 3887 & 23 & 16.76 \\
\hline & & Overall & 2147 & 2513 & 2240 & 2267 & 2484 & 2764 & 2364 & 16779 & 100 & 72.33 \\
\hline
\end{tabular}

Notes: BPR = birth prevalence rate calculated with 2,319,737 births in the 7 years analyzed; BPR by 10,000 total births. 
In Ecuador, every year 7339 children are admitted with a birth defect or other previously acquired complications; 18 patients out of every 10,000 hospitalizations are admitted with a congenital defect. Cardiac defects are the main reason for hospitalization and cleft lip is the most prevalent birth defect. Microtia and anotia show the highest prevalence in Latin America, according to the ECLAMC data. ${ }^{4}$ One percent of all deaths are from congenital malformations and $36 \%$ of these were mainly due to cardiac congenital defects.

Table 2 shows the birth defects in children 1 to 5 years of age. In this group the most common birth defect was unde- scended testicle with a BPR of 9.36/10,000 births, followed by unilateral cleft palate with a BPR of 5.32, patent ductus arteriosus with a BPR of 2.97 and unilateral cleft palate with a BPR of 2.72. This table reports 14,495 discharges in this particular group. Both analyzed groups comprise $60.7 \%(31,174$ admissions) of total admissions. The overall BPR of birth defects in children under 1 year is $55.58 / 10,000$ births and in children between 1 and 5 years is 50.33/10,000 births.

Table 3 compares prevalence rates by country and network; our prevalence rates are significantly lower than those of each country analyzed. Table 4 shows the major

Table 2 The most common birth defects in children I to 5 years of age that were admitted to Ecuadorian hospitals

\begin{tabular}{|c|c|c|c|c|c|c|c|c|c|c|c|c|}
\hline Rank & Code & Description & 2001 & 2002 & 2003 & 2004 & 2005 & 2006 & 2007 & Total & $\%$ & BPR \\
\hline I & Q539 & Undescended testicle, unspecified & 352 & 320 & 240 & 293 & 288 & 339 & 339 & $217 \mid$ & 14.98 & 9.36 \\
\hline 2 & Q359 & Cleft palate, unilateral & 188 & 223 & 173 & 167 & $|4|$ & 184 & 159 & 1235 & 8.52 & 5.32 \\
\hline 3 & Q250 & Patent ductus arteriosus & 92 & 116 & 75 & 102 & 94 & 88 & 121 & 688 & 4.75 & 2.97 \\
\hline 4 & Q369 & Cleft lip, unilateral & 120 & 114 & 68 & 82 & 97 & 78 & 73 & 632 & 4.36 & 2.72 \\
\hline 5 & Q38I & Ankyloglossia & 46 & 63 & 64 & 61 & 62 & 90 & 121 & 507 & 3.50 & 2.19 \\
\hline 6 & Q652 & Congenital luxation of the hip & 76 & 50 & 71 & 84 & 45 & 52 & 71 & 449 & 3.10 & 1.94 \\
\hline 7 & Q205 & Discordant atrioventricular connection & 63 & 106 & 56 & 44 & 36 & 43 & 52 & 400 & 2.76 & 1.72 \\
\hline 8 & Q039 & Congenital hydrocephalus & 63 & 0 & 64 & 53 & 43 & 77 & 76 & 376 & 2.59 & 1.62 \\
\hline 9 & Q658 & Other congenital deformities of hip & 18 & 28 & 22 & 25 & 33 & 72 & 68 & 266 & 1.84 & 1.15 \\
\hline 10 & Q249 & Congenital malformation of heart, unspecified & 33 & 30 & 39 & 36 & 41 & 30 & 49 & 258 & 1.78 & 1.11 \\
\hline II & Q660 & Talipes equinovarus & 9 & 51 & 53 & 26 & 24 & 34 & 55 & 252 & 1.74 & 1.09 \\
\hline 12 & Q379 & Unspecified cleft palate with unilateral cleft lip & 32 & 35 & 41 & 32 & 33 & 49 & 28 & 250 & 1.72 & 1.08 \\
\hline 13 & Q213 & Tetralogy of Fallot & 30 & 54 & 42 & 25 & 17 & 28 & 52 & 248 & 1.71 & 1.07 \\
\hline 14 & Q549 & Hypospadias & 38 & 50 & 24 & 32 & 22 & 39 & 40 & 245 & 1.69 & 1.06 \\
\hline 15 & Q699 & Polydactily & 41 & 33 & 27 & 27 & 40 & 44 & 28 & 240 & 1.66 & 1.03 \\
\hline 16 & Q663 & Other congenital varus deformities of feet & 26 & 21 & 31 & 37 & 35 & 19 & 35 & 204 & 1.41 & 0.88 \\
\hline 17 & Q892 & Persistent thyroglossal duct, thyroglossal cyst & 39 & 32 & 26 & 22 & 21 & 25 & 38 & 203 & 1.40 & 0.88 \\
\hline 18 & Q668 & $\begin{array}{l}\text { Deformities of feet: clubfoot, hammer toe, } \\
\text { congenital talipes }\end{array}$ & 30 & 36 & 14 & 30 & 28 & 36 & 22 & 196 & 1.35 & 0.84 \\
\hline 19 & Q909 & Down's syndrome & 21 & 35 & 26 & 36 & 28 & 21 & 27 & 194 & 1.34 & 0.84 \\
\hline 20 & Q750 & Craniosynostosis & 18 & 24 & 29 & 28 & 28 & 26 & 24 & 177 & 1.22 & 0.76 \\
\hline 21 & Q210 & Ventricular septal defect & 12 & 16 & 16 & 26 & 14 & 30 & 36 & 150 & 1.03 & 0.65 \\
\hline 22 & Q899 & Congenital malformation, unspecified & 5 & 0 & 31 & 67 & 12 & 24 & 8 & 147 & 1.01 & 0.63 \\
\hline 23 & Q439 & Congenital malformation of intestine, unspecified & 14 & 21 & 19 & 28 & 17 & 23 & 20 & 142 & 0.98 & 0.61 \\
\hline 24 & Q043 & Other reduction deformities of brain & 7 & 77 & 9 & 7 & 10 & 12 & 8 & 130 & 0.90 & 0.56 \\
\hline 25 & Q552 & $\begin{array}{l}\text { Other congenital malformations of testis } \\
\text { and scrotum }\end{array}$ & 27 & 15 & 17 & 13 & 10 & 19 & 29 & 130 & 0.90 & 0.56 \\
\hline 26 & Q43I & Hirschsprung's disease & 18 & 24 & 19 & 14 & 17 & 18 & 11 & 121 & 0.83 & 0.52 \\
\hline 27 & Q02 & Microcephaly & 14 & 10 & 14 & 23 & 15 & 28 & 17 & 121 & 0.83 & 0.52 \\
\hline 28 & Q709 & Syndactyly & 20 & 18 & 17 & 10 & 15 & 19 & 18 & 117 & 0.81 & 0.50 \\
\hline 29 & Q21I & Atrial septal defect & 7 & 13 & 12 & 14 & 9 & 18 & 39 & 112 & 0.77 & 0.48 \\
\hline 30 & Q532 & Undescended testicle, bilateral & 17 & 7 & 11 & 4 & 15 & 16 & 34 & 104 & 0.72 & 0.45 \\
\hline 31 & Q53I & Undescended testicle, unilateral & 0 & 0 & 1 & 1 & 1 & 10 & 86 & 99 & 0.68 & 0.43 \\
\hline 32 & Q740 & Other congenital malformations of limb(s) & 9 & 8 & 16 & 12 & 20 & 11 & 15 & 91 & 0.63 & 0.39 \\
\hline 33 & Q100 & Congenital blepharoptosis & 9 & 8 & 8 & 14 & 21 & 15 & 15 & 90 & 0.62 & 0.39 \\
\hline 34 & Q556 & Other congenital malformations of penis & 5 & 3 & 3 & 23 & 35 & 7 & 5 & 81 & 0.56 & 0.35 \\
\hline
\end{tabular}

(Continued) 
Table 2 (Continued)

\begin{tabular}{|c|c|c|c|c|c|c|c|c|c|c|c|c|}
\hline Rank & Code & Description & 2001 & 2002 & 2003 & 2004 & 2005 & 2006 & 2007 & Total & $\%$ & BPR \\
\hline 35 & Q423 & Imperforate anus & 22 & 13 & 11 & 3 & 8 & 12 & 9 & 78 & 0.54 & 0.34 \\
\hline 36 & QI8I & Preauricular sinus and cyst & 3 & 12 & 9 & 13 & 8 & 6 & 13 & 64 & 0.44 & 0.28 \\
\hline 37 & Q059 & Spina bifida, unspecified & 8 & 16 & 8 & 5 & 13 & 0 & 12 & 62 & 0.43 & 0.27 \\
\hline 38 & Q643 & $\begin{array}{l}\text { Other atresia and stenosis of urethra and } \\
\text { bladder neck }\end{array}$ & 10 & 23 & 6 & 5 & 1 & 5 & 11 & 61 & 0.42 & 0.26 \\
\hline 39 & Q393 & Congenital stenosis and stricture of esophagus & 15 & 7 & 6 & 5 & 8 & 10 & 6 & 57 & 0.39 & 0.25 \\
\hline 40 & Q620 & Congenital hydronephrosis & 3 & 5 & 5 & 7 & 8 & 11 & 16 & 55 & 0.38 & 0.24 \\
\hline $4 I$ & Q204 & Double inlet ventricle & 12 & 8 & 6 & 4 & 6 & 12 & 6 & 54 & 0.37 & 0.23 \\
\hline 42 & Q430 & Meckel's diverticulum & 6 & 12 & 3 & 5 & 10 & 11 & 7 & 54 & 0.37 & 0.23 \\
\hline 43 & $\mathrm{Q} 212$ & Atrioventricular septal defect & 10 & 8 & 7 & 5 & 5 & 4 & 10 & 49 & 0.34 & 0.21 \\
\hline 44 & Q172 & Microtia & 4 & I & 10 & 8 & 8 & 4 & 13 & 48 & 0.33 & 0.21 \\
\hline 45 & Q25 I & Coarctation of aorta & 4 & 8 & 3 & 3 & 5 & 9 & 16 & 48 & 0.33 & 0.21 \\
\hline 46 & Q54I & Hypospadias & I & 10 & 13 & 6 & 3 & 5 & 9 & 47 & 0.32 & 0.20 \\
\hline 47 & Q692 & Accessory toe(s) & 7 & 5 & 12 & 4 & 4 & 4 & 9 & 45 & 0.31 & 0.19 \\
\hline 48 & Q378 & Unspecified cleft palate with bilateral cleft lip & 2 & 4 & 14 & 8 & 5 & 3 & 7 & 43 & 0.30 & 0.19 \\
\hline 49 & Q540 & Hypospadias, balanic & 3 & 8 & 3 & 6 & 7 & 6 & 10 & 43 & 0.30 & 0.19 \\
\hline \multirow[t]{4}{*}{50} & Q255 & Atresia of pulmonary artery & 5 & 6 & 10 & 1 & 4 & 8 & 8 & 42 & 0.29 & 0.18 \\
\hline & & Subtotal & 1614 & 1787 & 1504 & 1586 & 1470 & 1734 & 1981 & 11676 & 80.5 & 50.33 \\
\hline & & Remain birth defects & 355 & 328 & 353 & 339 & 474 & 463 & 507 & 2819 & 19.5 & 12.15 \\
\hline & & Overall & 1969 & 2115 & 1857 & 1925 & 1944 & 2197 & 2488 & 14495 & 100 & 62.49 \\
\hline
\end{tabular}

Notes: BPR = birth prevalence rate calculated with 2'319,737 births in the 7 years analyzed.

congenital malformations reported by the ECLAMC Network, a rate calculated as 66,843 births out of 12 hospitals in this network.

Birth defects caused 1\% of all deaths in Ecuador during the period analyzed. Cardiac malformations $(35.95 \%$ of all cases) were the highest cause of birth defect mortality, followed by congenital malformations $(10.66 \%)$. Congenital malformations of spleen, adrenal gland, parathyroid or thyroid gland, persistent thyroglossal duct and cyst, and the situs inversus comprised the other unclassified causes (Table 5).

\section{Discussion}

\section{Birth defects}

The overall prevalence at birth of the selected congenital defects in Ecuador was $72.3 / 10,000$ births. This result is significantly lower than in the registries of other countries mainly due to under-reporting and the low quality of Ecuador's surveillance system. These figures should be taken as minimal estimates with uncertain diagnostic accuracy because Ecuador does not have an official medical birth registry or a congenital malformations registry.

However, in Ecuador the low level of education and cultural misconceptions have led many families to hide birth defects because their reporting could lead to the families being stigmatized by their social group, mainly in isolated rural populations. ${ }^{30}$ There is limited knowledge of genetics and its terminology in the general population. Shamanic and marginal health practices seem to remain prevalent especially in rural communities due to the lower costs and the lack of access to health care, the personal attention the individuals receive, and the holistic point of view used. ${ }^{31}$ Furthermore, there has been an increase in teenage pregnancies in recent years in Ecuador, aggravated by the major risk factors such as low family income, poor education, lack of knowledge about reproductive health and poor psychological family support. Adverse outcomes in mothers, such as high rates of cesarean section, puerperal infections, and complication during childbirth, have been identified; and adverse outcomes also in the fetus, such as premature birth, low birth weight and small size for gestational age have been identified also. All of these contribute to the numbers of birth defects. ${ }^{32}$

Because we found a massive under-reporting of birth defects in the Admissions/Discharge Registries, they are not a reliable single source for ascertaining congenital malformations. However, their availability allowed us to perform a natural experiment to assess our registration system and identify weaknesses in data collection. Our findings agreed with those of Penchaszadeh ${ }^{13}$ who noted that the birth prevalence of congenital anomalies in the developing world is underestimated by deficiencies in diagnostic capabilities and 
Table 3 Comparison of prevalence rates by country and network ${ }^{41}$

\begin{tabular}{|c|c|c|c|c|c|c|c|c|c|}
\hline Country & Latin-America & Cuba & Costa Rica & Mexico & Spain & Finland & Texas & Ecuador & Ecuador \\
\hline Network & ECLAMC & RECUMAC & CREC & RYVEMCE & ECEMC & CREC & BDES & ECLAMC & Our data* \\
\hline Year & 2005 & 2005 & 2005 & 2005 & 2005 & 2005 & 2004 & 2005 & 2009 \\
\hline Total births & 192,882 & I I 7,923 & 71,548 & 29,463 & 106,728 & 57,927 & 383,192 & 66,843 & $2,319,737$ \\
\hline Anencephaly & 5.08 & 2.46 & 1.26 & 2.72 & 0.19 & 3.11 & 2.24 & 3.1 & 0.34 \\
\hline Spina bifida & 8.71 & 5.43 & 2.80 & 4.75 & 1.50 & 5.01 & 4.49 & 4.2 & 2.49 \\
\hline Encephalocele & 2.64 & 1.36 & 0.56 & 1.02 & 0.28 & 2.07 & 0.55 & 0.9 & 0.48 \\
\hline Microcephaly & 3.94 & 0.93 & 1.68 & 3.05 & 1.03 & 1.73 & 8.72 & 1.9 & 0.59 \\
\hline Holoprosencephaly & 1.56 & 0.76 & 0.00 & 2.72 & 0.47 & 1.73 & 0.99 & $\mathrm{nr}$ & 0.04 \\
\hline Hydrocephaly & 14.78 & 8.90 & 1.26 & 7.47 & 1.97 & 5.18 & 6.03 & 5.5 & 3.77 \\
\hline Anophthalmos & 2.70 & 0.17 & 0.00 & 1.36 & 0.09 & 0.35 & 0.29 & $\mathrm{nr}$ & $\mathrm{nr}$ \\
\hline Microphthalmos & 1.30 & 0.17 & 0.70 & $\mathrm{nr}$ & $\mathrm{I} .4 \mathrm{I}$ & 1.21 & 2.30 & $\mathrm{nr}$ & $\mathrm{nr}$ \\
\hline $\begin{array}{l}\text { Anophthalmos/ } \\
\text { microphthalmos }\end{array}$ & 0.00 & 0.08 & 0.00 & $\mathrm{nr}$ & 0.00 & 0.00 & 0.00 & 2.1 & $\mathrm{nr}$ \\
\hline Anotia & 0.41 & 0.08 & 0.00 & 9.16 & 0.00 & $\mathrm{nr}$ & 0.31 & $\mathrm{nr}$ & $\mathrm{nr}$ \\
\hline Microtia & 6.95 & 0.25 & 1.96 & $\mathrm{nr}$ & 1.31 & $\mathrm{nr}$ & 3.00 & 10.8 & 1.19 \\
\hline Unspecified anotia/microtia & 0.10 & 0.25 & 0.00 & $\mathrm{nr}$ & 0.00 & 4.66 & 0.00 & $\mathrm{nr}$ & $\mathrm{nr}$ \\
\hline Transposition of great vessels & 0.62 & 2.37 & 0.14 & 0.68 & 1.22 & 3.63 & 4.36 & 2.1 & 0.43 \\
\hline Tetralogy of Fallot & 1.24 & 1.53 & 1.12 & 0.00 & 0.75 & 4.66 & 2.66 & $\mathrm{nr}$ & 0.64 \\
\hline $\begin{array}{l}\text { Hypoplastic left heart } \\
\text { syndrome }\end{array}$ & 1.04 & 1.53 & 0.14 & 0.00 & 0.28 & 4.49 & 2.01 & 0.3 & $\mathrm{nr}$ \\
\hline Coarctation of aorta & 0.31 & 1.10 & 0.28 & 0.00 & 0.75 & 8.11 & 4.38 & $\mathrm{nr}$ & 0.19 \\
\hline Choanal atresia, bilateral & 0.21 & 0.00 & 0.28 & 0.00 & 0.09 & 1.04 & 0.73 & $\mathrm{nr}$ & 0.17 \\
\hline Cleft palate without cleft lip & 5.86 & 2.37 & 2.38 & 3.05 & 4.12 & 14.33 & 5.09 & 4.2 & 1.91 \\
\hline $\begin{array}{l}\text { Cleft lip with or without } \\
\text { cleft palate }\end{array}$ & 14.52 & 4.66 & 6.01 & 12.56 & 3.09 & 11.05 & 11.46 & 18 & 3.24 \\
\hline $\begin{array}{l}\text { Esophageal atresia/stenosis } \\
\text { with or without fistula }\end{array}$ & 3.37 & 2.37 & 2.38 & 2.72 & 1.87 & 3.80 & 1.57 & 2.8 & 0.93 \\
\hline Small intestine atresia/stenosis & 3.99 & 1.87 & 0.98 & 2.38 & 0.37 & 0.86 & 1.72 & 0.9 & 0.62 \\
\hline Anorectal atresia/stenosis & 5.81 & 0.93 & 3.77 & 4.41 & 1.97 & 4.66 & 4.33 & 3.9 & 1.23 \\
\hline Undescended testis & 7.05 & 3.48 & 8.39 & $\mathrm{nr}$ & 2.44 & $\mathrm{nr}$ & 10.52 & $\mathrm{nr}$ & 1.21 \\
\hline Hypospadias & 4.15 & 10.18 & 4.89 & 5.77 & 1.31 & 12.08 & 16.26 & 2.5 & 0.18 \\
\hline Epispadias & 0.05 & 0.34 & 0.14 & 0.34 & 0.19 & 2.76 & 0.65 & $\mathrm{nr}$ & $\mathrm{nr}$ \\
\hline Indeterminate sex & 2.49 & 0.51 & 1.82 & 2.72 & 0.66 & 0.00 & 0.81 & $\mathrm{nr}$ & 0.08 \\
\hline Renal agenesis & 2.64 & 0.85 & 1.12 & 1.02 & 0.19 & 1.55 & 1.91 & $\mathrm{nr}$ & 0.05 \\
\hline Cystic kidney & 3.27 & 2.80 & 0.28 & 0.68 & 1.78 & 1.90 & 5.30 & 2.2 & 0.31 \\
\hline Bladder exstrophy & 0.21 & 0.17 & 0.00 & 0.34 & 0.09 & 7.60 & 0.29 & $\mathrm{nr}$ & 0.03 \\
\hline Polydactyly, preaxial & 4.25 & 0.85 & 9.50 & 11.20 & 3.19 & 0.69 & 3.00 & 3 & 1.11 \\
\hline Diaphragmatic hernia & 3.63 & 2.46 & 2.80 & 1.36 & 1.03 & 0.00 & 2.56 & 1.6 & 0.47 \\
\hline Omphalocele & 4.51 & 2.37 & 1.54 & 2.04 & 0.84 & 3.80 & 2.04 & 1.3 & 0.38 \\
\hline Gastroschisis & 5.18 & 3.31 & 1.12 & 5.77 & 0.09 & 5.35 & 4.18 & 0.1 & 0.78 \\
\hline Prune belly sequence & 0.73 & 0.08 & 0.56 & 0.68 & 0.09 & 0.17 & 0.31 & $\mathrm{nr}$ & $\mathrm{nr}$ \\
\hline Trisomy 13 & 0.67 & 1.19 & 1.12 & 0.00 & 0.47 & 0.35 & 1.17 & $\mathrm{nr}$ & $\mathrm{nr}$ \\
\hline Trisomy 18 & 1.71 & 1.36 & 1.82 & 0.00 & 0.47 & 1.38 & 2.45 & $\mathrm{nr}$ & 0.05 \\
\hline Down's syndrome, all ages & $|8.5|$ & 10.94 & 8.11 & 11.20 & 7.40 & 7.60 & 12.81 & 13.6 & 3.70 \\
\hline
\end{tabular}

*Our data are from the national Registry of Admission/Discharge, source INEC." It analyzed the data from children under I year of age.

Abbreviations: ECLAMC, Latin American Collaborative Study of Congenital Malformations; RECUMAC, Cuban Register of Congenital Malformation; RYVEMCE, Mexican Registry and Epidemiological Surveillance of External Congenital Malformations; ECEMC, Spanish Collaborative Study of Congenital Malformations; BDES, Texas Birth Defects Epidemiology and Surveillance Branch; CREC, Costa Rican Birth Defects Register Center. ${ }^{41}$

lack of reliability of medical records and health statistics. As a result, recorded diagnoses in statistics focus on overt acute illnesses, rather than on pre-existing congenital conditions that increase vulnerability to infections and malnutrition.
Cleft lip was the most prevalent birth defect in children under 1 year of age, and the second most common defect in children of 1 to 5 years of age. This finding seems similar to that of the Poletta ${ }^{33}$ study, who said that for cleft lip and cleft 
Table 4 Major congenital malformations in Ecuador reported by the ECLAMC Network ${ }^{4}$

\begin{tabular}{|c|c|c|c|c|c|}
\hline Malformation & $\mathbf{N}=$ & Rate & ICD-I 0 & ICD-8 & Rank \\
\hline Cleft lip & 120 & 18 & Q35 & $749 \mathrm{~A}$ & 1 \\
\hline Down's syndrome & 91 & 13.6 & Q90 & 7593 & 2 \\
\hline Postaxial polidactily & 90 & 13.5 & Q69 & 7550 & 3 \\
\hline Talipes equinovarus & 73 & 10.9 & Q66 & 7541 & 4 \\
\hline Microtia/anotia & 72 & 10.8 & Q17 & $745 \mathrm{~A}$ & 5 \\
\hline Subluxation of hip & 72 & 10.8 & Q65 & 7556 & 6 \\
\hline Hydrocephaly & 37 & 5.5 & Q03 & 7420 & 7 \\
\hline Bifid spine & 28 & 4.2 & Q05 & $74 I$ & 8 \\
\hline Cleft palate & 28 & 4.2 & Q36 & 7490 & 9 \\
\hline Imperforate anus & 26 & 3.9 & Q42 & 7512 & 10 \\
\hline Anencephaly & 21 & 3.1 & Q00 & 7400 & 11 \\
\hline Septal defects & 20 & 3.0 & Q21 & 746SE & 12 \\
\hline Preaxial polidactily & 20 & 3.0 & Q69 & $7550 \mathrm{~B}$ & 13 \\
\hline Esophageal atresia & 19 & 2.8 & Q39 & 7502 & 14 \\
\hline Syndactyly & 18 & 2.7 & Q70 & $755 I R$ & 15 \\
\hline Hypospadias & 17 & 2.5 & Q54 & 7522 & 16 \\
\hline Limb agenesia (amputation) & 17 & 2.1 & Q74 & $755 / 2$ & 17 \\
\hline Polycystic kidney & 15 & 2.2 & Q61 & 7531 & 18 \\
\hline Trunk conal defect & 14 & 2.1 & Q21 & 746TO & 19 \\
\hline Anophthalmos/microphthalmos & 14 & 2.1 & QII & 744 & 20 \\
\hline Talipes talovalgus & 13 & 1.9 & Q66 & 7542 & 21 \\
\hline Microcephaly & 13 & 1.9 & Q02 & 7431 & 22 \\
\hline Congenital hydronephrosis & 12 & 1.8 & Q62 & 7532 & 23 \\
\hline Diaphragmatic hernia & II & 1.6 & Q79 & 75681 & 24 \\
\hline Other cardiopathies & 10 & 1.5 & Q20 & 746 & 25 \\
\hline
\end{tabular}

Notes: BPR calculated from 66,843 lives births in 12 hospitals of the network. Abbreviations: ICD, International Classification of Diseases 10th Edition and 8th Edition. ${ }^{29}$

palate high birth prevalence rates clusters were associated with high altitude above sea level, Native Amerindian ancestry, and low socioeconomic status; low birth prevalence rate clusters showed an association with African and Black ancestry. Advanced maternal age is a recognized risk factor for cleft palate only. Weinberg ${ }^{34}$ has suggested that similar occult lip defects are present in individuals affected with isolated cleft palate, and also that cleft palate and cleft lip plus cleft palate are etiologically distinct. These findings raise the possibility that some cleft palate cases may be misclassified.

Facial clefts are a heterogeneous group of easy-torecognize, nonfatal birth defects. Worldwide, it was reported as the most common congenital facial abnormality. A small proportion occur as a part of recognizable patterns of malformations or a genetic etiology, while epidemiologic data suggest that exogenous factors contribute to these conditions. Maternal factors, which have been studied for their influence on cleft risk, include smoking, alcohol consumption, medication use, environmental chemicals and nutritional
Table $\mathbf{5}$ General mortality rates by birth defects in Ecuador

\begin{tabular}{|c|c|c|c|}
\hline Year & $\mathbf{N}=$ & PMR & $\begin{array}{l}\text { Mortality } \\
\text { rate }\end{array}$ \\
\hline 2001 & 580 & 1.050 & 0.045 \\
\hline 2002 & 510 & 0.918 & 0.039 \\
\hline 2003 & 480 & 0.897 & 0.037 \\
\hline 2004 & 503 & 0.919 & 0.039 \\
\hline 2005 & 555 & 0.977 & 0.042 \\
\hline 2006 & 617 & 1.065 & 0.046 \\
\hline 2007 & 678 & 1.169 & 0.050 \\
\hline Total & 3923 & 1.001 & 0.043 \\
\hline ICD-10 & $\mathbf{N}=$ & & $\%$ \\
\hline $\begin{array}{l}\text { Q24: Dextrocardia, levocardia, cor } \\
\text { triatriatum, pulmonary infundibular } \\
\text { stenosis, congenital subaortic stenosis, } \\
\text { malformation of coronary vessels, } \\
\text { congenital heart block, others }\end{array}$ & 919 & & 23.43 \\
\hline $\begin{array}{l}\text { Q89: Congenital malformations of spleen, } \\
\text { of adrenal gland, other endocrine glands; } \\
\text { situs inversus; conjoined twins; multiple } \\
\text { congenital malformations }\end{array}$ & 418 & & 10.66 \\
\hline Q03: Congenital hydrocephalus & 271 & & 6.91 \\
\hline $\begin{array}{l}\text { Q0I: Anencephaly and similar } \\
\text { malformations }\end{array}$ & 204 & & 5.20 \\
\hline $\begin{array}{l}\text { Q21: Congenital malformations } \\
\text { of cardiac septa }\end{array}$ & 204 & & 5.20 \\
\hline $\begin{array}{l}\text { Q25: Congenital malformations } \\
\text { of great arteries }\end{array}$ & 172 & & 4.38 \\
\hline Q90: Down's syndrome, trisomy 21 & 157 & & 4.00 \\
\hline $\begin{array}{l}\text { Q20: Congenital malformations } \\
\text { of cardiac chambers and connections }\end{array}$ & 115 & & 2.93 \\
\hline $\begin{array}{l}\text { Q79: Congenital diaphragmatic hernia, } \\
\text { exomphalos, gastroschisis, prune belly } \\
\text { syndrome, others }\end{array}$ & 112 & & 2.85 \\
\hline $\begin{array}{l}\text { Q87: Multisystemic birth defects, } \\
\text { congenital syndromes }\end{array}$ & 110 & & 2.80 \\
\hline Subtotal & 1763 & & 68.37 \\
\hline Others birth defects & 2160 & & 31.63 \\
\hline Overall & 3923 & & 100.00 \\
\hline $\begin{array}{l}\mathrm{Q} 20+\mathrm{Q} 21+\mathrm{Q} 24+\mathrm{Q} 25 \\
\text { (all cardiac birth defects) }\end{array}$ & 1410 & & 35.94 \\
\hline
\end{tabular}

Source: INEC."

Abbreviation: PMR, proportionate mortality rate.

factors, but none appear to explain a significant proportion of the population burden of these anomalies. Geographic differences in birth prevalence for these anomalies probably reflect differences in maternal life style and exposure to environmental causative factors.

Undescended testicle was the most prevalent birth defect in children between 1 and 5 years of age. Cryptorchidism is a common congenital anomaly that shows familial clustering and increased prevalence in first-degree relatives, 
suggesting that genetic factors contribute to the etiology. Nonsyndromic cryptorchidism is a common and complex disorder of unknown etiology with geographic and, perhaps, temporal variability. Although presumed to be multifactorial in etiology, few specific genetic or environmental factors have been clearly linked to this disease; there is evidence for multilocus genetic susceptibility. ${ }^{35}$

Cardiovascular system defects were by far the most common group of birth defects in our population. The prevalence rate for this kind of defect was very similar to rates previously published in comparable countries. ${ }^{36}$ However, it was possibly underdiagnosed in some pathologies due to lack of routine diagnostic systems, and because echocardiography and karyotype analysis are unavailable in the smaller provinces. It should be stressed that small cardiac defects close spontaneously in $30 \%$ to $50 \%$ of the cases. The most common cardiac birth defect was patent ductus arteriosus or patent arterial duct (PAD), defined as persistent patency in term infants older than 3 months. Isolated PAD is found worldwide in around 1 out of 2000 full-term infants. A higher prevalence is found in preterm infants, especially those of low birth weight. ${ }^{37}$ Persistence of the duct is associated with chromosomal aberrations, asphyxia at birth, birth at high altitude and congenital rubella. Occasional cases are associated with specific genetic defects (trisomy 21 and 18, and the RubinsteinTaybi and CHARGE syndromes). Familial occurrence of PAD is uncommon and the usual mechanism of inheritance is considered to be polygenic, with a recurrence risk of $3 \%$. Rare families with isolated PAD have been described in whom the inheritance appears to be dominant or recessive. ${ }^{37}$

Over the past few decades, there has been major progress in the understanding and management of cardiac birth defects, including identification of genetic causes for some conditions. Although early interventions have transformed the outcome for these patients, many have ongoing problems that require tertiary cardiac care in adult life. Furthermore, most adults require additional nontertiary care for issues such as pregnancy, noncardiac surgery, endocarditis and other problems. It is an important problem in public health, especially in relation to the costs of the hospitalization. These findings indicate a strong need to standardize both diagnostic and registration criteria for congenital heart malformations.

Our overall figure for Down's syndrome was 3.8/10,000 births. Other published population-based rates for this syndrome are higher, as the ECLAMC study ${ }^{4}$ that found a BPR of 13.6/10,000 births. Down's can be diagnosed relatively easily prior to birth by measuring alphafetoprotein, human chorionic gonadotropin and unconjugated estriol in fetal serum, detecting a thickened nuchal fold on fetal ultrasound, and by cytogenetic analysis. Thus, it is likely that many fetuses with Down's were aborted electively and that variable abortion rates contribute to the variable prevalence of birth rates. In Ecuador, abortion by professional clinicians is allowed.

The prevalence of specific types of congenital anomalies at low and high altitudes in South America is higher in the high altitude lands than in the lowlands, especially for six types of birth defects: cleft lip, microtia, preauricular tag, branchial arch anomaly complex, constriction band complex, and anal atresias. ${ }^{38}$ Other authors have shown that altitude is associated with low birth weight and intrauterine growth retardation. ${ }^{39}$ These could be the most likely explanations ${ }^{40}$ for the unilateral cleft lip, the most frequent birth defect in Ecuador.

\section{Limitations and strengths of this study}

This report has at least five limitations in relation with the findings. First, this report does not determine severity within each type of birth defect; it analyzed the crude data without analyzing in depth each clinical situation. This limits the utility of these data for health-care planning, and justifies broader studies on this issue.

Second, this analysis could not separate children with isolated defects from those with more than one major defect or those with a recognized syndrome because the used registries do not emphasize these defects, and training in and knowledge of genetics of the health care providers, especially in primary care, is still very limited.

Third, it was not possible to determine how much of the variation is attributable to specific risk factors, and the prevalence of some of the defects is influenced by the sources of prenatal diagnoses used; this prevalence varies across hospitals and provinces in Ecuador. In some cases, the limitations are greater and clinical experience still remains the main criterion of diagnosis. The findings in this report represent a conservative estimate of the number of cases each year across the nation, but these data are only an estimate of congenital defects, which would be higher for some defects, if the data were based on systems that included prenatal and postnatal sources.

Fourth, these national estimates represent minimum estimates for the impact of these defects, because even those surveillance systems with active case-findings do not achieve $100 \%$ ascertainment.

Finally, some of the most common birth defects could be under represented and could not be included because identification of these conditions depends on referral patterns and access to and use of diagnostic procedures which vary from one hospital to another. 


\section{The creation of a National Registry of Birth Defects}

This report documents the prevalence estimates for birth defects reported in the Hospital Discharge data. These estimates are important to 1) plan for health-care and education needs of the Ecuadorian population, 2) identify increased occurrence of birth defects in specific geographic regions, 3) serve as a reference point for assessment of provincial surveillance systems, 4) evaluate national public health interventions, 5) compare Ecuador prevalence estimates with those of other countries, and 6) help determine the appropriate allocation of resources for basic and public health research.

Hospital-based estimates help quantify the public health importance of these defects and can help improve the planning of services for affected children and their families; eg, the need for specific clinical specialists or multispecialty clinics. Children affected by certain birth defects could benefit from the availability of multispecialty clinics to address the coordination of multiple needs and continuity of ongoing care in a single setting. Because not all provinces have surveillance systems, the national prevalence estimates can be used by provinces to assess health services and special education programs, as well as to evaluate and improve their existing health programs and ensure that affected children are referred to the appropriate services.

Accurate national prevalence estimates of birth defects are essential because birth defects are one of the leading causes of infant mortality and a major contributor to childhood morbidity. Continued improvement can be achieved by a) enhancing completeness of ascertainment, b) increasing the consistency of methods among birth defects surveillance systems, and c) expanding the methods of data collection and analysis of birth defects for which reliable and valid estimates of national prevalence can be made.

In general, this study was limited to selected major birth defects, even though it is the first to provide population-based data on the prevalence rates within their class. The methods used for data collection and case generation are not enough to provide all the required information. Comparison with an independent source such as the birth medical history would be necessary to complete and improve the quality of the registries. Also, it would be important to begin registering defects in elective abortions, and in private medical offices where pathological prenatal identification is routine. As life can be sustained with many malformations, particularly with modern treatment and surgical correction, they are not detected by vital registration systems. Mortality data cannot provide reliable indications of birth prevalence of live birth malformations, and thus registries of such malformations are important.

Birth defects are a true public health problem that has been underestimated by health authorities in Ecuador. The emphasis by public health professionals on solving simpler problems such as diarrhea and respiratory infections has meant the neglect of this group of diseases, and birth defects have become almost hidden diseases. This study allows us to understand the urgent need to establish a National Registry of Birth Defects, involving different sources of information such as prenatal medical records, birth records, and postnatal medical records during the first year of life at an early stage, and surveys of cytogenetic prenatal diagnostic surveys and cytogenetics of therapeutic abortions.

The demonstrated prevalence and lack of documentation are sufficient reasons to initiate this registry. Of course, this new record will have to be combined with other measures such as a genetics education program for health providers, a public campaign to encourage healthcare workers to report cases of patients with genetic defects, and a specialized genetic counseling training program.

\section{Contribution of authors}

The conception, study design analysis and interpretation of data, drafting, critical revising and final approval of this manuscript were perfomed equally by FGA and RLP. Both authors are the guarantors of the paper.

\section{Ethical approval}

Not required as the study concerned retrospective analysis of a database.

\section{Disclosures}

The authors declare no conflicts of interest. No funding was received for this work.

\section{References}

1. Penchaszadeh VB, Beiguelman B. Medical genetics services in Latin America: report of a meeting of experts. Rev Panam Salud Publica/Pan Am J Public Health. 1998;3:409-420.

2. Monitoring Birth Defects. http://www.cdc.gov/ncbddd/bd/monitoring. htm. Accessed April 9, 2009.

3. Castilla EE, López-Camelo JS. The surveillance of birth defects in South America: I, The search for time clusters: epidemics. Adv Mutagen Res. 1990;2:191-210.

4. Montalvo G, Giron C, Camacho A, Martinez E, Toscano M, et al. Frecuencia de malformaciones congénitas en hospitales Ecuatorianos de la red ECLAMC. Período junio 2001-junio 2005. Revista Cambios, 5 (9) 2005. [In Spanish] http://www.prenatal.tv/lecturas/ecuador/ ECLAMC_Montalvo.pdf. Accessed Accessed April 9, 2009.

5. Castilla EE, Sod R. The surveillance of birth defects in South America. II, The search for geographic clusters: endemics. Adv Mutagen Res. 1990;2:211-230. 
6. Cunha AJB. Orientação genética-clínica em medicina fetal. In: Isfer EV, Sanchez RC, Saito M, organizadores. Medicina fetal diagnóstico prénatal e conduta. Rio de Janeiro: Revinter; 1996:1-19.

7. Han A, Rotermann M, Fuller-Thomson E, Ray JG. Pre-conceptional folic acid supplement use according to maternal country of birth. J Obstet Gynaecol Can. 2009;31:222-226.

8. González-Andrade F, López-Pulles R. Ecuador: Public Health Genomics. Public Health Genomics. 2009. DOI: 10.1159/000249817.

9. United States. The CIA World Factbook 2008. Washington, DC: Central Intelligence Agency, 1900s. https:/www.cia.gov/library/publications/ the-world-factbook/geos/ec.html. Accessed April 9, 2009.

10. Kold Jensen T. Congenital malformations. In: Children's health and environment: A review of evidence. A joint report from the European Environment Agency and the WHO Regional Office for Europe. Office for Official Publications of the European Community; 2002:116-126.

11. INEC, Instituto Ecuatoriano de Estadísticas y Censos. Integrated System of Electronic Consults, National Vital Statistics. http://www.inec. gov.ec/. Accessed April 9, 2009.

12. González-Andrade F, Sánchez D, Martínez-Jarreta B. Sex-specific genetic admixture of Mestizos. Amerindian Kichwas and Blacks Afroamericans from Ecuador (South America). Human Biol. 2007;78:51-78.

13. Penchaszadeh V. Preventing congenital anomalies in developing countries. Community Genet. 2002;5:61-69.

14. Canfield MA, Ramadhani TA, Shaw GM, et al; National Birth Defects Prevention Study. Anencephaly and spina bifida among Hispanics: maternal, sociodemographic, and acculturation factors in the National Birth Defects Prevention Study. Birth Defects Res A Clin Mol Teratol. 2009;85:637-646.

15. Centers for Disease Control and Prevention. Update on Overall Prevalence of Major Birth Defects-Atlanta, Georgia, 1978-2005. Morb Mort Weekly Report. 2008;57:1-5.

16. EUROCAT, Network of Anomaly Registers. http://www.eurocat.ulster ac.uk. Accessed April 9, 2009.

17. Hobbs CA, Cleves MA, Simmons CJ. Genetic epidemiology and congenital malformations: From the chromosome to the crib. Arch Pediatr Adolesc Med. 2002;156:315-320.

18. Yoon PW, Rasmussen SA, Lynberg MC, et al; and The National Birth Defects Prevention Study. The National Birth Defects Prevention Study: Methods. Public Health Reports. 2001;116(Suppl 1):32-40.

19. Lie RT, Wilcox AJ, Skjærven R. Survival and reproduction among males with birth defects and risk of recurrence in their children. JAMA. 2001;285:755-760.

20. Tilford JM, Robbins JM, Hobbs CA. Improving estimates of caregiver time cost and family burden associated with birth defects. Teratology. 2001;64(S1):37-41.

21. Rasmussen SA, Erickson JD, Reef SE, Ross DS. Teratology: from science to birth defects prevention. Birth Defects Res A Clin Mol Teratol. 2009;85:82-92.

22. Mulholland C, Njoroge T, Mersereau P, Williams J. Comparison of guidelines available in the United States for diagnosis and management of diabetes before, during, and after pregnancy. $J$ Womens Health (Larchmt). 2007;16:790-801.

23. Corrigan N, Brazil DP, McAuliffe F. Fetal cardiac effects of maternal hyperglycemia during pregnancy. Birth Defects Res A Clin Mol Teratol. 2009;85:523-530.
24. Morice A, Ulloa-Gutierrez R, Avila-Agüero ML. Congenital rubella syndrome: progress and future challenges. Expert Rev Vaccines. 2009;8:323-331.

25. Ornoy A. Neuroteratogens in man: an overview with special emphasis on the teratogenicity of antiepileptic drugs in pregnancy. Reprod Toxicol. 2006;22:214-226.

26. Meaney FJ, Miller LA; FASSNet Team. A comparison of fetal alcohol syndrome surveillance network and birth defects surveillance methodology in determining prevalence rates of fetal alcohol syndrome. Birth Defects Res A Clin Mol Teratol. 2003;67:819-822.

27. Floyd RL, Weber MK, Denny C, O'Connor MJ. Prevention of fetal alcohol spectrum disorders. Dev Disabil Res Rev. 2009;15: 193-199.

28. MSP, Ministry of Public Health of Ecuador, National Epidemiological Surveillance System. http://www.msp.gov.ec/index.php?option=com_c ontent\&task=blogsection\&id=21\&Itemid=175. Accessed April 9, 2009.

29. WHO, International Statistical Classification of Diseases and Related Health Problems, 10th Revision, Version for 2007, Chapter XVII: Congenital malformations, deformations and chromosomal abnormalities (Q00-Q99). http://www.who.int/classifications/apps/icd/icd10online/. Accessed April 9, 2009.

30. Vivar SC. Ecuador addresses cultural issues for pregnant women. Lancet. 2007;370:1302.

31. Paz-Y-Miño C, Sánchez ME, Sarmiento I, Leone PE. Genetics and congenital malformations: interpretations, attitudes and practices in suburban communities and the shamans of Ecuador. Community Genet. 2006;9:268-273.

32. Chedraui P. Pregnancy among young adolescents: trends, risk factors and maternal-perinatal outcome. J Perinat Med. 2008;36: 256-259.

33. Poletta FA, Castilla EE, Orioli IM, López-Camelo JS. Regional analysis on the occurrence of oral clefts in South America. Am J Med Genet A. 2007;143A:3216-3227.

34. Weinberg SM, Brandon CA, McHenry TH, et al. Rethinking isolated cleft palate: evidence of occult lip defects in a subset of cases. Am J Med Genet A. 2008;146A:1670-1675.

35. Chacko JK, Barthold JS. Genetic and environmental contributors to cryptorchidism. Pediatr Endocrinol Rev. 2009;6:476-480.

36. Baltaxe E, Zarante I. Prevalence of congenital heart disease in 44,985 newborns in Colombia. Arch Cardiol Mex. 2006;76:263-268.

37. Forsey JT, Elmasry OA, Martin RP. Patent arterial duct. Orphanet $J$ Rare Dis. 2009;4:17.

38. Castilla EE, Lopez-Camelo JS, Campaña H. Altitude as a risk factor for congenital anomalies. Am J Med Genet. 1999;86:9-14.

39. Webster WS, Howe AM, Abela D, Oakes DJ. The relationship between cleft lip, maxillary hypoplasia, hypoxia and phenytoin. Curr Pharm Des. 2006;12:1431-1448.

40. Moore LG. Fetal growth restriction and maternal oxygen transport during high altitude pregnancy. High Alt Med Biol. 2003;4: $141-156$.

41. International Clearinghouse For Birth Defects Surveillance and Research (ICBDSR), Annual Report with data for 2005. The ICBDSR Centre, Roma, Italy, 2007

The Application of Clinical Genetics

\section{Publish your work in this journal}

The Application of Clinical Genetics is an international, peer-reviewed open access journal that welcomes laboratory and clinical findings in the field of human genetics. Specific topics include: Population genetics; Functional genetics; Natural history of genetic disease; Management of genetic disease; Mechanisms of genetic disease; Counselling and

\section{Dovepress}

ethical issues; Animal models; Pharmacogenetics; Prenatal diagnosis; Dysmorphology. The manuscript management system is completely online and includes a very quick and fair peer-review system, which is all easy to use. Visit http://www.dovepress.com/testimonials.php to read real quotes from published authors. 